\title{
Healthcare Costs Incurred by Patients with Multiple Myeloma Following Triple Class Exposure (TCE) in the US
}

Sundar Jagannath $\cdot$ Nedra Joseph $\cdot$ Jinghua He $\cdot$ Concetta Crivera

Alex Z. Fu · Ashraf Garret $\cdot$ Nina Shah

Received: September 17, 2021 / Accepted: October 5, 2021 / Published online: October 25, 2021

(C) The Author(s) 2021

\section{ABSTRACT}

Introduction: Multiple myeloma $(\mathrm{MM})$ is a malignancy of plasma cells; most MM patients will eventually relapse or become refractory to treatment. Treating MM patients remains a challenge since patients eventually progress through several lines of therapy (LOTs), requiring the use of multiple MM drug classes. We examined healthcare resource utilization (HCRU) and the costs incurred by MM patients following triple class exposure (TCE; defined as exposure to a proteosome inhibitor, an

Supplementary Information The online version contains supplementary material available at https:// doi.org/10.1007/s40487-021-00175-z.

S. Jagannath

Icahn School of Medicine at Mount Sinai, New York, NY, USA

e-mail: sundar.jagannath@mountsinai.org

N. Joseph $(\bowtie) \cdot C$. Crivera

Real World Value and Evidence (Oncology), Janssen

Scientific Affairs, LLC, Horsham, PA, USA

e-mail: PJoseph3@ITS.JNJ.com

C. Crivera

e-mail: CCrivera@its.jnj.com

J. He - A. Z. Fu

Janssen Scientific Affairs, LLC, Titusville, NJ, USA

J. He

e-mail: JHe31@ITS.JNJ.com immunomodulatory agent, and an anti-CD-38 antibody).

Methods: Adult MM patients were selected from the MarketScan ${ }^{\circledR}$ commercial and Medicare supplemental databases (January 2009-February 2021). From this cohort, patients who had TCE and $\geq 1$ subsequent LOT that occurred after January 1, 2017 were included in the study population. The initiation date for the first postTCE LOT was defined as the index date. Allcause and MM-related HCRU and the associated costs were examined post-index date.

Results: A total of $85 \mathrm{MM}$ patients with TCE who initiated $\geq 1$ subsequent LOT post-TCE and had $\geq 1$ year of follow-up post-index date

\author{
A. Z. Fu \\ Georgetown University Medical Center, \\ Washington, DC, USA \\ e-mail: ZFu@ITS.JNJ.com

\section{A. Garret} \\ Legend Biotech Inc., Piscataway, NJ, USA \\ e-mail: Ashraf.Garrett@legendbiotech.com \\ N. Shah \\ University of California San Francisco, San \\ Francisco, CA, USA \\ e-mail: Nina.Shah@ucsf.edu
}


were included in the study population; mean age on index date was 58.8 years, and $60.0 \%$ were male. The time from first-observed MM diagnosis until index date averaged 47.5 months. During an average follow-up of 20.9 months post-index date, $64.7 \%$ of patients $(N=55)$ initiated a second LOT and $35.2 \%$ $(N=30)$ received at least 3 LOTs. During followup, mean total all-cause healthcare cost per patient was $\$ 722,992$ (equivalent to $\$ 34,578$ per patient per month [PPPM]). Approximately $90.7 \%$ (\$655,524 per patient) of the total allcause healthcare costs were MM related, $66.0 \%$ of which were MM drug/infusion costs.

Conclusion: In this real-world US study, MM patients with TCE incurred high healthcare costs, with the majority being MM related and primarily attributed to $\mathrm{MM}$ drug and infusion costs.

Keywords: Healthcare costs; Healthcare resource utilization; Multiple myeloma; Triple class exposure

\section{Key Summary Points}

Why carry out this study?

Multiple myeloma (MM) is a malignancy of plasma cells and accounts for approximately $10 \%$ of all hematological cancers and $1.8 \%$ of all newly diagnosed cancer cases in the United States.

Most patients with this cancer will eventually relapse or become refractory to treatment. Thus, treating patients with MM remains a challenge, as these patients eventually progress through several lines of therapy (LOTs).
To gain a better understanding of the potential value of new therapies for treating MM, we utilized a large US administrative healthcare claims database to examine healthcare resource utilization and the costs incurred by MM patients following triple class exposure (TCE; defined as exposure to a proteosome inhibitor, an immunomodulatory agent, and an anti-CD-38 antibody) during January 2017 through February 2021.

\section{What was learned from the study?}

During an average follow-up of 20.9 months, the mean total all-cause healthcare cost per patient was $\$ 722,992$ (equivalent to $\$ 34,578$ per patient per month [PPPM]), of which the majority was MM related and primarily attributed to $\mathrm{MM}$ drug and infusion costs.

These study findings underscore the significant need to develop and make widely available other novel targeted therapies for the treatment of MM patients to potentially improve patient outcomes and reduce the healthcare economic burden of this patient population.

\section{INTRODUCTION}

Multiple myeloma (MM) is a malignancy of plasma cells and accounts for approximately $10 \%$ of all hematological cancers and $1.8 \%$ of all newly diagnosed cancer cases in the United States [1, 2]. In 2021 in the US, it is estimated that 34,920 new cases of MM will be diagnosed, and 12,410 deaths will be attributed to the disease $[1,3]$. The median age at diagnosis is 69 years, males (new cases: 8.8 per 100,000) are more commonly affected than females (new cases: 5.7 per 100,000), and non-Hispanic Blacks have twice the incidence of Whites and other races [1, 3].

Since the late 1970s, the 5- to 6-year survival rate of MM patients has more than doubled, from 25 to $55 \%[4,5]$. This improvement in 
patient outcomes has in part been attributed to the introduction of several drug classes of MM therapies in the last few decades, including proteasome inhibitors (PIs; bortezomib, carfilzomib, and ixazomib), immunomodulatory drugs (IMiD; lenalidomide, thalidomide, and pomalidomide), targeted monoclonal antibodies (daratumumab, elotuzumab, and isatuximab), selective inhibitor of exportin-1 (selinexor), and the BCMA-targeting antibody-drug conjugate belantamab mafodotin. Despite widespread utilization of these therapies for the treatment of $\mathrm{MM}$, the disease remains incurable, and most patients will eventually relapse or become refractory to treatment $[4,6]$. As a result, treating patients with MM remains a challenge, as these patients eventually progress through several lines of therapy (LOTs). Currently used MM treatments in LOTs comprise doublet and triplet regimens containing multiple drug classes, a principal recommendation for the treatment of $\mathrm{MM}$ patients in the National Cancer Comprehensive Network (NCCN) 2021 guidelines [7].

There are several novel targeted therapies currently in development for the treatment of relapsed/refractory multiple myeloma (RRMM), and the treatment landscape is expected to rapidly evolve in the near future [4]. To gain a better understanding of the potential value of new therapies for treating patients with RRMM, who have triple class exposure (TCE) and limited treatment options, it is important to have a current assessment of the healthcare resource utilization (HCRU) and economic burden for these patients. Towards this aim, we utilized a large US administrative healthcare claims database to examine HCRU and the costs incurred by $\mathrm{MM}$ patients with commercial insurance coverage following TCE, defined as exposure to a PI, IMiD, and anti-CD-38 antibody, during January 2017 through February 2021.

\section{METHODS}

\section{Study Design and Data Source}

This study was a retrospective observational cohort study that utilized the IBM ${ }^{\circledR}$
MarketScan ${ }^{\circledR}$ Commercial Claims and Encounters (CCAE) and Medicare Supplemental (MDCR) databases. The CCAE database is a fully adjudicated, paid medical and pharmacy insurance administrative healthcare claims database of over 130 million unique persons, including active employees, early retirees, COBRA continuers, and their dependents insured by employer-sponsored health plans. The MDCR database is an administrative healthcare claims database for Medicare-eligible active and retired employees and their Medicare-eligible dependents from employer-sponsored supplemental plans. Both databases capture utilization and costs of inpatient and outpatient medical services and pharmacy services as well as patient demographics and enrollment status. Used primarily for research and containing only deidentified patient dataset, the CCAE and MDCR databases are fully compliant with the Health Insurance Portability and Accountability Act (HIPAA).

\section{Study Population}

The patients included in this study were adults ( $\geq 18$ years of age) diagnosed with MM between January 1, 2009 and February 28, 2021. MM was identified according to the International Classification of Diseases (ICD) 9th/10th Revision Clinical Modification codes, ICD-9: $203.0 \times$ and ICD-10: C90.0x. Patients were required to have received at least one NCCN guideline-recommended $\mathrm{MM}$ treatment [6] (Supplementary Table 1) on or after their first observed MM diagnosis date, and to be continuously enrolled in a medical/pharmacy benefit plan for at least 12 months prior to their firstobserved MM diagnosis. Patients diagnosed with other types of malignancies prior to their first-observed MM diagnosis dates were excluded from the study population.

From this overall MM patient cohort, we identified the subset of patients who had TCE, defined as exposure to a PI, an IMiD, and an anti-CD38 targeted antibody, during treatment following the first-observed $\mathrm{MM}$ diagnosis. To estimate costs post-TCE, we also required that these patients have at least one subsequent LOT 
post-TCE. The initiation date of the first LOT post-TCE was defined as the index date for patients in the TCE cohort. Post-TCE LOTs were required to have occurred after January 1, 2017, in order to capture contemporary HCRU and cost estimates. Furthermore, using survival duration after TCE as a proxy measure for "fitness" in this MM population who have already cycled through multiple LOTs, patients were required to have survived at least 1 year after their index date. Patients were followed until the end of the study period, continuous enrollment, or death, whichever occurred first.

\section{Definitions of Treatment Regimens and LOTs}

Each regimen was defined by a start date, end date, and a distinct regimen made up of one or more drugs. A patient could have received multiple treatment regimens in sequence during the course of treatment. Every occurrence of a treatment change (including augmentation and switching) or initiation of a new regimen after discontinuation of a previous one constituted a LOT change.

A regimen was formed by one or several NCCN-recommended medications (Supplementary Table 1) for MM identified from both medical and pharmacy claims during the first 60 days following the regimen initiation. The index treatment date was the initiation date of the index regimen (i.e., the date of the first claim for an identified MM treatment). Each regimen ended on either the discontinuation date or the date of treatment change, whichever occurred first. The date of treatment change was the initiation date of the next LOT. Changes in any agent ('targeted' or chemotherapy) within 60 days of the first treatment was not considered a LOT change.

Gaps were calculated as the days elapsed between the last day of supply of a dispensing and its subsequent dispensing. The maximum allowed gap was 90 days in this study. If a stem cell transplant (SCT) occurred prior to the end of a gap, another 6-month allowed gap was added after the SCT.
A medication was considered discontinued if it was not refilled within the maximum allowed gap after the expiration of supply days of the last dispensing. If the medication was identified from a claim using a Healthcare Common Procedure Coding (HCPCS) code, the days of supply was imputed using the recommended treatment schedule.

An augmentation was defined as receiving a new medication of interest at least 60 days prior to discontinuing any of the medications included in the current regimen. The date of initiating the new medication was considered the augmentation date. A switch was defined as receiving a new medication of interest with at least one medication of the current regimen discontinued prior to or within 60 days after the initiation of the new medication. The initiation date of the new medication was the switch date. The addition of chemotherapy agents (e.g., cyclophosphamide, doxorubicin, melphalan) constituted a LOT change.

A regimen was discontinued when all medications included in the regimen were discontinued or a treatment change occurred (including augmentation and switching). If only a corticosteroid remained after discontinuation of other therapies in the regimen, that was considered a regimen discontinuation.

If a patient used a maintenance therapy (e.g., lenalidomide or bortezomib monotherapy) within 6 months after a SCT, regardless of whether lenalidomide or bortezomib was part of the initial regimen, this was not considered switching and did not trigger a LOT change. The discontinuation date for the regimen was extended to the discontinuation of the lenalidomide or bortezomib monotherapy.

\section{Demographic and Clinical Characteristics}

For each patient in the TCE study cohort, demographic and clinical characteristics, including age, sex, health plan type, insurance type (Commercial or Medicare), US region of residence, Quan Charlson Comorbidity Index (QCI) score, and year of index date, were evaluated on the patients' index dates or during the 12 months prior to index dates. Additionally, 
the time from first-observed MM diagnosis until index date was determined.

\section{HCRU and Associated Costs}

During the 12-month period prior to the patients' index dates, all-cause and MM-related (i.e., claims including an ICD-9/10 code indicating a MM diagnosis) HCRU, including the number of hospitalizations and days of inpatient stays, number of emergency room (ER) visits, number of outpatient visits, and number of pharmacy fills (all-cause only), were examined for all patients in the TCE study cohort. During the same period, all-cause and MM-related healthcare costs were also measured. MMrelated healthcare costs are reported with a breakdown for inpatient cost, ER cost, outpatient cost, drug costs, drug infusion cost, SCT cost, and other healthcare costs, all of which were reported as the average cost per patient.

For the primary analysis of this study, healthcare costs-all cause and MM relatedwere examined during the follow-up periods after a patient's index date (i.e., after the first post-TCE treatment initiation) and were reported as the average cost per patient and as per patient per month (PPPM); results were reported for the overall TCE cohort, as well as for patients $<65$ years (Commercially insured) who made up the vast majority $(89.4 \%)$ of the study sample. The average monthly total and MM-related healthcare costs per patient were also extrapolated in order to estimate the total costs incurred for an additional duration (in this case, up to 40 months after a patient's index date).

\section{Statistical Analyses}

All analyses conducted in this study were descriptive. Means and standard deviations were reported for continuous variables and numbers and percentages for categorical variables. All statistical analyses were carried out using SAS software, version 9.4 (SAS Institute Inc., Cary, North Carolina).
Table 1 Characteristics of patients who initiated $\geq 1$ LOT post-TCE

\begin{tabular}{|c|c|}
\hline & $N=85$ \\
\hline Age in, years, ${ }^{a}$ mean $(S D)$ & $58.8(7.6)$ \\
\hline \multicolumn{2}{|l|}{ Age group, $N(\%)$} \\
\hline $18-44$ & $3(3.5)$ \\
\hline $45-54$ & $18(21.2)$ \\
\hline $55-64$ & $55(64.7)$ \\
\hline $65-74$ & $5(5.9)$ \\
\hline$\geq 75$ & $4(4.7)$ \\
\hline \multicolumn{2}{|l|}{$\operatorname{Sex}, N(\%)$} \\
\hline Male & $51(60.0)$ \\
\hline Female & $34(40.0)$ \\
\hline \multicolumn{2}{|l|}{ Health plan type, $N$ (\%) } \\
\hline Comprehensive & $12(14.1)$ \\
\hline Health maintenance organization (HMO) & $9(10.6)$ \\
\hline Preferred provider organization (PPO) & $30(35.3)$ \\
\hline Other & $34(40.0)$ \\
\hline \multicolumn{2}{|l|}{ Insurance type, $N(\%)$} \\
\hline Commercial & $76(89.4)$ \\
\hline Medicare & $9(10.6)$ \\
\hline \multicolumn{2}{|l|}{ US region of residence, $N(\%)$} \\
\hline South & $39(45.9)$ \\
\hline Midwest & $21(24.7)$ \\
\hline West & $17(20.0)$ \\
\hline Northeast & $8(9.4)$ \\
\hline QCI score, mean (SD) & $3.5(3.1)$ \\
\hline \multicolumn{2}{|l|}{ Index date year, $N(\%)$} \\
\hline 2017 & $24(28.2)$ \\
\hline 2018 & $27(31.8)$ \\
\hline 2019 & $29(34.1)$ \\
\hline 2020 & $5(5.9)$ \\
\hline
\end{tabular}


Table 1 continued

\begin{tabular}{ll}
\hline & $N=\mathbf{8 5}$ \\
\hline $\begin{array}{l}\text { Time in months from first-observed MM } \\
\text { diagnosis to index date, mean (SD) }\end{array}$ & $47.5(26.4)$ \\
\hline
\end{tabular}

QCI Quan-Charlson Comorbidity Index score, SD standard deviation, TCE triple class exposure

a On index date

\section{RESULTS}

\section{Characteristics of the TCE Cohort}

A total of $85 \mathrm{MM}$ patients who initiated at least one subsequent LOT post-TCE and had at least 1 year of follow-up post-index date were included in the study population. Among this patient cohort, mean age as of the index date was 58.8 years, and $60.0 \%$ were male (Table 1 ). Approximately one-third $(35.3 \%)$ of patients had preferred provider organization (PPO) health plans (Table 1). Mean QCI score was 3.5 (Table 1).

The time from a first-observed MM diagnosis until index date averaged 47.5 months; the duration of the first LOT post-TCE was approximately 10.4 months.

\section{HCRU and Costs Prior to the Index Date}

During the 12 months prior to the index date there were, on average, 1.7 all-cause hospitalizations, $0.6 \mathrm{ER}$ visits, and 88.5 outpatient visits per patient (Table 2). The average length of hospitalization stay was 16.1 days (Table 2). Mean total all-cause healthcare costs per patient were $\$ 439,871$ (Table 2).

During the same 12-month period, there were 1.4 hospitalizations, 0.2 ER visits, and 69.9 outpatient visits per patient that were MM-related (Table 2). The average length of MM-related hospitalizations was 15.4 days (Table 2). Mean total MM-related healthcare costs per patient were $\$ 414,323$, which represented $94.2 \%$ of total all-cause healthcare costs; on
Table 2 HCRU and costs during the 12 months prior to the index date ${ }^{\mathrm{a}}$

All-cause HCRU, mean (SD)

Number of hospitalizations

$1.7(3.2)$

Length of inpatient stays in days

$16.1(30.8)$

Number of emergency room visits

$0.6(1.1)$

Number of outpatient visits

88.5 (107.7)

Number of pharmacy fills

42.5 (19.6)

All-cause total healthcare costs, mean

$\$ 439,871$

$(S D)^{\mathrm{b}}$

$(\$ 272,509)$

$M M$-related HCRU, mean (SD)

Number of hospitalizations

$1.4(2.7)$

Length of inpatient stays in days

$15.4(30.6)$

Number of emergency room visits

$0.2(0.4)$

Number of outpatient visits

$69.9(84.8)$

$M M$-related healthcare costs, mean $(S D)^{\mathrm{b}}$

Inpatient cost

$\$ 51,097$

Emergency room cost

$\$ 449(\$ 1,230)$

Outpatient cost

$\$ 58,850$

$(\$ 68,016)$

MM drug cost

$\$ 263,600$

$(\$ 170,639)$

MM drug infusion cost

$\$ 11,705$

$(\$ 12,004)$

Stem cell transplant cost

$\$ 24,893$

Other costs

$\$ 3,730(\$ 2,460)$

Total costs

$\$ 414,323$

$(\$ 260,961)$

$H C R U$ healthcare resource utilization, LOT line of therapy, $M M$ multiple myeloma, $S D$ standard deviation, TCE triple class exposure

a Index date, defined as the initiation date of first LOT post-TCE

${ }^{b}$ Average cost for all patients in the sample; patients may not have incurred a cost in every category 
Table 3 Total all-cause healthcare costs and MM-related healthcare costs incurred following the index date ${ }^{a}$

\begin{tabular}{|c|c|c|c|c|}
\hline & \multirow{3}{*}{$\begin{array}{l}\text { All } \\
\text { patients } \\
N=85\end{array}$} & \multirow{3}{*}{$\begin{array}{l}\text { Patients }<65 \text { years of } \\
\text { age (Commercially } \\
\text { insured) } \\
N=76\end{array}$} & \multicolumn{2}{|l|}{ following the index date ${ }^{a}$} \\
\hline & & & $\begin{array}{l}\text { Month post-TCE LOT } \\
\text { initiation }\end{array}$ & $\begin{array}{l}\text { All-cause total healthcare } \\
\text { costs }\end{array}$ \\
\hline & & & 1 & $\$ 34,578$ \\
\hline \multirow{4}{*}{$\begin{array}{l}\text { Follow-up duration in } \\
\text { months, mean } \\
\text { All-cause total } \\
\text { healthcare costs per } \\
\text { patient, mean }\end{array}$} & 20.9 & 21.1 & 2 & $\$ 69,156$ \\
\hline & \multirow{3}{*}{$\$ 722,992$} & \multirow{3}{*}{$\$ 749,503$} & 3 & $\$ 103,734$ \\
\hline & & & 4 & $\$ 138,312$ \\
\hline & & & 5 & $\$ 172,890$ \\
\hline \multirow{3}{*}{$\begin{array}{l}\text { All-cause total } \\
\text { healthcare costs } \\
\text { PPPM, mean }\end{array}$} & \multirow[t]{5}{*}{$\$ 34,578$} & \multirow[t]{5}{*}{$\$ 35,473$} & 6 & $\$ 207,468$ \\
\hline & & & 7 & $\$ 242,046$ \\
\hline & & & 8 & $\$ 276,624$ \\
\hline \multirow{2}{*}{$\begin{array}{l}\text { MM-related } \\
\text { healthcare costs per } \\
\text { patient, mean }\end{array}$} & & & 9 & $\$ 311,202$ \\
\hline & & & 10 & $\$ 345,780$ \\
\hline Inpatient cost & $\$ 89,668$ & $\$ 97,260$ & 11 & $\$ 380,358$ \\
\hline \multirow{2}{*}{$\begin{array}{l}\text { Emergency room } \\
\text { cost }\end{array}$} & \multirow[t]{2}{*}{$\$ 1,007$} & \multirow[t]{2}{*}{$\$ 918$} & 12 & $\$ 414,936$ \\
\hline & & & 13 & $\$ 449,514$ \\
\hline Outpatient cost & $\$ 105,076$ & $\$ 113,548$ & 14 & $\$ 484,092$ \\
\hline MM drug cost & $\$ 415,475$ & $\$ 419,288$ & 15 & $\$ 518,670$ \\
\hline \multirow{2}{*}{$\begin{array}{l}\text { MM drug infusion } \\
\text { cost }\end{array}$} & \multirow[t]{2}{*}{$\$ 17,405$} & \multirow[t]{2}{*}{$\$ 18,239$} & 16 & $\$ 553,248$ \\
\hline & & & 17 & $\$ 587,826$ \\
\hline $\begin{array}{l}\text { Stem cell transplant } \\
\text { cost }\end{array}$ & $\$ 21,123$ & $\$ 23,055$ & 18 & $\$ 622,404$ \\
\hline \multirow{2}{*}{$\begin{array}{l}\text { Other costs } \\
\text { Total costs }\end{array}$} & $\$ 5,770$ & \multirow[t]{2}{*}{$\$ 5,937$} & 19 & $\$ 656,982$ \\
\hline & $\$ 655,524$ & & 20 & $\$ 691,560$ \\
\hline \multirow{4}{*}{\multicolumn{3}{|c|}{$\begin{array}{l}{ }^{a} \text { Index date, defined as the initiation date of first LOT } \\
\text { post-TCE } \\
\text { Average cost for all patients in the sample; patients may } \\
\text { not have incurred a cost in every category } \\
L O T \text { line of therapy, } M M \text { : multiple myeloma, PPPM per } \\
\text { patient per month, TCE triple class exposure }\end{array}$}} & 22 & $\$ 760,716$ \\
\hline & & & 23 & $\$ 795,294$ \\
\hline & & & 24 & $\$ 829,872$ \\
\hline & & & 25 & $\$ 864,450$ \\
\hline & & & 26 & $\$ 899,028$ \\
\hline & & & 27 & $\$ 933,606$ \\
\hline \multirow{4}{*}{\multicolumn{3}{|c|}{$\begin{array}{l}\text { average, of the total MM-related healthcare } \\
\text { costs per patient, inpatient hospitalizations } \\
\text { accounted for } 12.3 \% \text {, outpatient visits } 14.2 \% \text {, } \\
\text { and MM drug and infusion costs } 66.4 \% \\
\text { (Table 2). }\end{array}$}} & 28 & $\$ 968,184$ \\
\hline & & & 29 & $\$ 1,002,762$ \\
\hline & & & 30 & $\$ 1,037,340$ \\
\hline & & & 31 & $\$ 1,071,918$ \\
\hline
\end{tabular}

Table 4 Extrapolation of average monthly all-cause healthcare costs to estimate cumulative costs incurred following the index date ${ }^{a}$ 
Table 4 continued

\begin{tabular}{ll}
\hline $\begin{array}{l}\text { Month post-TCE LOT } \\
\text { initiation }\end{array}$ & $\begin{array}{l}\text { All-cause total healthcare } \\
\text { costs }\end{array}$ \\
\hline 32 & $\$ 1,106,496$ \\
33 & $\$ 1,141,074$ \\
34 & $\$ 1,175,652$ \\
35 & $\$ 1,210,230$ \\
36 & $\$ 1,244,808$ \\
37 & $\$ 1,279,386$ \\
38 & $\$ 1,313,964$ \\
39 & $\$ 1,348,542$ \\
40 & $\$ 1,383,120$ \\
\hline
\end{tabular}

LOT line of therapy, TCE triple class exposure

a Index date, defined as the initiation date of first LOT post-TCE

Italicized costs are projected based on the dataset of patients included in this study with an average follow-up of 20.9 months

\section{HCRU and Costs After Index Date}

The average duration of follow-up post-index date was 20.9 months; $64.7 \% \quad(N=55)$ of patients initiated a second LOT post-TCE and $35.2 \%(N=30)$ received $\geq 3$ LOTs. Over this follow-up period, the mean total all-cause healthcare costs per patient were $\$ 722,992$ (equivalent to $\$ 34,578$ PPPM). Among patients $<65$ years of age, the mean total allcause healthcare costs per patient were $\$ 749,503$ (equivalent to $\$ 35,473$ PPPM).

During patient follow-up, approximately $90.7 \%(\$ 655,524$ per patient) of the total allcause healthcare costs were MM related, and most $(66.0 \%)$ of these costs were attributed to MM drug and infusion costs (Table 3). Among patients $<65$ years of age, mean total MM-related healthcare costs per patient were $\$ 678,245$; $90.5 \%$ of the total all-cause healthcare costs per patient were MM related (Table 3 ).
The average patient follow-up duration in this study was approximately 21 months. This is related to restricting the index date for post-TCE treatments to 2017 onwards to capture contemporary HCRU and associated costs. Therefore, we also extrapolated the total all-cause healthcare costs for a period of up to 40 months after the index date. When extrapolating beyond the average follow-up period of patients in this study, the cumulative all-cause healthcare costs incurred per patient at 24 months post-index date was estimated at $\$ 829,872$, and at 36 months was estimated at $\$ 1,244,808$ (Table 4 ).

\section{DISCUSSION}

This real-world study of MM patients in the US with TCE provides a contemporary view of the healthcare costs incurred by this patient population as they progress through multiple LOTs. During the follow-up period of 20.9 months, the mean total all-cause healthcare cost per patient was $\$ 722,992$ (equivalent to $\$ 34,578$ PPPM), of which approximately $91 \%$ were MM related. Our study findings reflect more recent cost estimates (through February 2021) and are generally consistent with those reported in the study by Madduri et al. in which patients were also identified from the MarketScan CCAE and MDCR databases. In that study, $154 \mathrm{MM}$ patients were selected who had TCE and initiated at least one subsequent LOT between December 1, 2015 and September 30, 2018 [8]. Total all-cause healthcare costs were estimated at $\$ 37,033$ PPPM in the 6 months (median follow-up) after initiation of another LOT postTCE; MM-related healthcare costs represented $96 \%$ of total all-cause healthcare costs [8]. Also, similar to our study findings, over one-half of the monthly MM-related healthcare costs were attributed to MM drug and infusion costs.

The findings of our study also show that following TCE, MM patients continue to receive additional LOTs due to disease progression and accumulate more healthcare costs during their treatment journey. The economic burden of treating $\mathrm{MM}$ patients is also impacted by increasing trends in the incidence of $\mathrm{MM}$ in the US [3] and patients becoming refractory to 
currently used MM drugs [6]. Triplet regimens containing IMiDs, PIs, and/or anti-CD38 targeted antibodies have been shown in clinical trials to induce more sustained clinical responses and progression-free survival compared to other MM treatments [9] and are recommended as the standard of care [7]. Additionally, newer medications (i.e., pomalidomide, carfilzomib, elotuzumab, daratumumab, ixazomib, and the bortezomib-lenalidomide combination) for the treatment of RRMM have demonstrated improved effectiveness over older therapies in a real-world US study [10]. However, the clinical benefits are diminished as patients continue to progress through multiple LOTs with shorter intervals between sequential LOTs, highlighting the unmet need in this patient population [10].

As the treatment landscape evolves for MM patients, it will be important to put into context the substantial cumulative healthcare economic burden associated with currently used MM treatments to better understand the potential value of newer therapies with novel mechanisms of action. Additionally, it will be necessary to take into consideration that these patients will often relapse and become refractory to currently used MM treatments, especially the accumulated clinical burden of patient exposure to multiple MM drug classes and the progression to increasingly resistant disease, which is ultimately indicative of worse patient outcomes $[6,10,11]$.

This study was a retrospective observational cohort study that utilized the MarketScan CCAE and MDCR databases, and the study findings should be interpreted from the perspective of certain limitations. First, the number of $\mathrm{MM}$ patients with TCE who received at least one subsequent LOT post-TCE was small in this study, and future follow-up studies are warranted. The population included in this analysis represented those patients with Commercial insurance and/or employer-sponsored Medicare coverage, and the study findings may not be generalizable to MM patients with other insurance types. In this study, approximately $11 \%$ of the overall study population were $\geq 65$ years of age, and further study is needed utilizing other data sources that may have higher representations of $\mathrm{MM}$ patients in this age group.
Additionally, due to the disproportionate regional distribution across the US of patient administrative claims contained within the data sources used for this study, other studies in specific US regions, as well as in other countries, may be needed to more fully understand the healthcare costs of MM patients with TCE.

Administrative healthcare claims filed for reimbursement may have contained erroneous coding and misdiagnoses, but likely to only a small extent due to the adjudication of the MarketScan databases. As it was an observational study, only associations between exposure variables and outcomes could be established, not causal inferences. The prescriptions identified in the claims databases reflected those filled by patients, and may not have captured all that were actually prescribed. Lastly, the treatments assessed in this study were approved at different times, and the utilization of drug combinations continued to evolve.

\section{CONCLUSIONS}

In this real-world US study, we found that the overall healthcare costs incurred by $\mathrm{MM}$ patients with TCE were high, averaging $\$ 34,578$ PPPM, of which the majority was MM related and primarily attributed to MM drug and infusion costs. These study findings underscore the significant need to develop and make widely available other novel targeted therapies for the treatment of $\mathrm{MM}$ patients to potentially improve patient outcomes and reduce the healthcare economic burden of this patient population.

\section{DATA AVAILABILITY STATEMENT}

All data generated or analyzed during this study are included in this published article. 


\section{ACKNOWLEDGEMENTS}

Funding. Sponsorship for this study and Rapid Service Fee were funded by Janssen Scientific Affairs, LLC.

Medical writing and/or editorial assistance. Medical writing and editorial assistance in the preparation of this article were provided by Melissa Lingohr-Smith and Jay Lin of Novosys Health and was funded by Janssen Scientific Affairs, LLC. Shayan Ghosh and Nagendra Chauhan of Mu Sigma, LLC provided scientific programming support.

Authorship. All named authors meet the International Committee of Medical Journal Editors (ICMJE) criteria for authorship for this article, take responsibility for the integrity of the work as a whole, and have given their approval for this version to be published.

Authorship contributions. All named authors contributed to concept, design, and data interpretation. Jinghua He and Alex Fu contributed to statistical analyses. All named authors contributed to the drafting of the manuscript.

Disclosures. Sundar Jagannath has been or is a consultant to Bristol Myers Squibb, Janssen Pharmaceuticals, Karyopharm Therapeutics, Legend Biotech, Sanofi, and Takeda. Nedra Joseph, Jinghua He, Concetta Crivera, and Alex $\mathrm{Z} \mathrm{Fu}$ are employees of Janssen Scientific Affairs, LLC and own company stock. Ashraf Garret is an employee of Legend Biotech and owns company stock. Nina Shah has been or is a consultant to GlaxoSmithKline, Amgen, Indapta Therapeutics, Sanofi, Bristol Myers Squibb, CareDx, Kite, and Karyopharm, and has received research funding from Celgene/Bristol Myers Squibb, Janssen, Bluebird Bio, Sutro Biopharma, Teneobio, and Poseida.

Compliance with ethics guidelines.. The data sources used in this study (i.e., the IBM ${ }^{\circledR}$ MarketScan ${ }^{\circledR}$ Commercial Claims and Encounters (CCAE) and Medicare Supplemental
(MDCR) databases) contain de-identified patient dataset and are fully compliant with the Health Insurance Portability and Accountability Act (HIPAA).

Data availability statement. All data generated or analyzed during this study are included inthis published article.

Open Access. This article is licensed under a Creative Commons Attribution-NonCommercial 4.0 International License, which permits any non-commercial use, sharing, adaptation, distribution and reproduction in any medium or format, as long as you give appropriate credit to the original author(s) and the source, provide a link to the Creative Commons licence, and indicate if changes were made. The images or other third party material in this article are included in the article's Creative Commons licence, unless indicated otherwise in a credit line to the material. If material is not included in the article's Creative Commons licence and your intended use is not permitted by statutory regulation or exceeds the permitted use, you will need to obtain permission directly from the copyright holder. To view a copy of this licence, visit http://creativecommons.org/licenses/bync/4.0/.

\section{REFERENCES}

1. National Cancer Institute: Surveillance, Epidemiology, and End Results Program. Cancer stat facts: myeloma. Available at: https://seer.cancer.gov/ statfacts/html/mulmy.html. Accessed 10 Jun 2021.

2. Michels TC, Peterson KE. Multiple myeloma: diagnosis and treatment. Am Fam Physician. 2017;95: 373-383A.

3. American Cancer Society: Cancer Statistics Center. Myeloma. Available at: https://cancerstatistics center.cancer.org/\#!/cancer-site/Myeloma. Accessed 10 Jun 2021.

4. Bobin A, Liuu E, Moya N, et al. Multiple myeloma: an overview of the current and novel therapeutic approaches in 2020. Cancers (Basel). 2020;12:2885.

5. Wong R, Tay J. Economics of multiple myeloma. Blood. 2018;132:4773. 
6. Pinto V, Bergantim R, Caires HR, Seca H, Guimaraes JE, Vasconcelos MH. Multiple myeloma: available therapies and causes of drug resistance. Cancers (Basel). 2020;12:407.

7. National Comprehensive Cancer Network. NCCN clinical practice guidelines in oncology: Multiple myeloma. Version 7.2021, April 26, 2021. Plymouth Meeting: National Comprehensive Cancer Network; 2021.

8. Madduri D, Hagiwara M, Parikh K, et al. Real-world treatment patterns, healthcare use and costs in triple-class exposed relapsed and refractory multiple myeloma patients in the USA. Future Oncol. 2021;17:503-15.
9. Landgren O, Iskander K. Modern multiple myeloma therapy: deep, sustained treatment response and good clinical outcomes. J Intern Med. 2017;281: 365-82.

10. Bruno AS, Wilson JL, Opalinska JM. Recent realworld treatment patterns and outcomes in US patients with relapsed/refractory multiple myeloma. Expert Rev Hematol. 2020;13:1017-25.

11. Yong K, Delforge M, Driessen C, et al. Multiple myeloma: patient outcomes in real-world practice. Br J Haematol. 2016;175:252-64. 